\title{
Miastenia Gravis Juvenil, atuação fisioterapêutica e desospitalização: relato de caso
}

\author{
Juvenile Myasthenia Gravis, physiotherapeutic approach, \\ and de-hospitalization: a case report
}

\author{
Miastenia Gravis Juvenil, actuación fisioterápica y \\ deshospitalización: informe de un caso
}

\author{
Ana Paula Jesus Pereira dos Santos ${ }^{1}$, Deusiane Santos Victor ${ }^{2}$, \\ Tatiane Regina Oliveira Santos ${ }^{1}$, Andressa Franco Moreira ${ }^{1}$, Luanna \\ Andrade Jacob Rebelo ${ }^{1}$, Nilo Manoel Pereira Vieira Barreto ${ }^{3}$, \\ Michelli Christina Magalhães Novais ${ }^{4}$
}

\begin{abstract}
1.Acadêmica em Fisioterapia, Centro Universitário Jorge Amado (UNIJORGE). Salvador-BA, Brasil.
2.Fisioterapeuta. Especialista. Fisioterapeuta no Hospital Martagão Gesteira (HMG) e Hospital Ana Nery (HAN). Salvador-BA, Brasil.

3.Enfermeiro. Mestre. Doutorando no Programa de Pós-graduação em Processos Interativos dos Órgãos e Sistemas (PPgPIOS), Universidade Federal da Bahia (UFBA). Salvador-BA, Brasil.

4.Fisioterapeuta. Mestre. Doutoranda no Programa de Pós-graduação em Processos Interativos dos Órgãos e Sistemas (PPgPIOS), Universidade Federal da Bahia (UFBA). Professora no Centro Universitário Jorge Amado (UNIJORGE). Salvador-BA, Brasil.
\end{abstract}

\begin{abstract}
Resumo
Objetivo. Descrever a atuação fisioterapêutica e a evolução funcional de uma criança com Miastenia Gravis Juvenil (MGJ). Método. Estudo observacional, descritivo, longitudinal, retrospectivo, de caso único: relato de caso; desenvolvido em hospital pediátrico de referência, em Salvador, Bahia, Brasil, através de dados de prontuário, registrados pela equipe multiprofissional de saúde, sobre a evolução clínica e funcional da criança. Resultados. Paciente MRS deu entrada em Unidade de Pronto Atendimento, com descompensação clínica, evoluiu para quadro de choque séptico e insuficiência respiratória aguda, levando à intubação orotraqueal. Foi transferida para Unidade de Terapia Intensiva, onde foi diagnosticada com pneumonia comunitária e após falhas na extubação, submetida à traqueostomia. Foi transferida para Unidade de Treinamento em Desospitalização (UTD), onde a fraqueza muscular persistente levou a investigação e diagnóstico de MGJ. Nesta unidade recebeu intervenções fisioterapêuticas com foco na atividade e participação, apresentou progressos no quadro motor, realizando ortostatismo independente, deambulação e subida/descida de escadas com suporte unilateral. Entretanto, permaneceu em ventilação mecânica invasiva. Conclusão. A atuação fisioterapêutica foi pautada na funcionalidade, visando capacidade e desempenho, utilizando-se da ludicidade como ferramenta para estimular a adesão ao tratamento. Como desfecho funcional, houve redução do grau de deficiências e limitações.
\end{abstract}

Unitermos. Miastenia Gravis; Fisioterapia; Pediatria; Relato de caso

\footnotetext{
Abstract

Objective. To describe the physiotherapeutic approach and the functional evolution of a child with Juvenile Myasthenia Gravis (JMG). Method. Observational, descriptive, longitudinal, retrospective, single-case study: case report; developed in a pediatric reference hospital in Salvador, Bahia, Brazil. The study was reliant upon medical record data about the clinical and functional evolution of the child and recorded by a multi-professional health team. Results. Patient MRS was admitted to the Emergency Care Unit with clinical decompensation. The patient developed septic shock and acute respiratory failure, which necessitated orotracheal intubation. The patient was transferred to the Intensive Care Unit, where she was diagnosed with Community-Acquired Pneumonia. After extubation failure, the
} 
patient underwent tracheostomy. The patient was transferred to the Dehospitalization Training Unit (DTU), where persistent muscle weakness led to the investigation and diagnosis of JMG. In this unit, the patient received physical therapy interventions which focused on activity and participation. The patient showed progress in motor framework - performing independent orthostatism, walking and climbing/descending stairs with unilateral support. However, the patient remained on invasive mechanical ventilation. Conclusion. The physiotherapeutic approach was based on functionality, aiming capacity and performance, using playfulness as a tool to encourage adherence to treatment. As a functional outcome, there was a reduction in the degree of disabilities and limitations.

Keywords. Myasthenia Gravis; Physical therapy; Pediatrics; Case report

\section{Resumen}

Objetivo. Describir la actuación fisioterápica y la evolución funcional de una niña con Miastenia Gravis Juvenil (MGJ). Método. Estudio observacional, descriptivo, longitudinal, retrospectivo, de caso único: informe de un caso; desarrollado en un hospital pediátrico de referencia, en Salvador, Bahía, Brasil, mediante datos de prontuario, registrados por el equipo multiprofesional de salud, respecto a la evolución clínica y funcional de la niña. Resultados. La paciente M.R.S. fue ingresada en una Unidad de Urgencias, con descompensación clínica, evolucionó a un cuadro de choque séptico e insuficiencia respiratoria aguda, lo que ocasionó su intubación orotraqueal. Fue transferida a una Unidad de Terapia Intensiva, donde se la diagnosticó con neumonía adquirida en la comunidad y, tras fallas en la extubación, fue sometida a una traqueostomía. Se la transfirió a una Unidad de Entrenamiento en Deshospitalización (UTD), donde la debilitación muscular persistente ocasionó la averiguación y diagnóstico de MGJ. Recibió en tal unidad intervenciones fisioterápicas con énfasis en la actividad y participación, presentó progresos en su cuadro motor, con la realización de ortostatismo independiente, deambulación y subida/bajada de escaleras con soporte unilateral. Sin embargo, permaneció con ventilación mecánica invasiva. Conclusión. La acción fisioterapéutica se basó en la funcionalidad, apuntando a la capacidad y al rendimiento, utilizando la alegría como herramienta para favorecer la adherencia al tratamiento. Como resultado funcional, hubo una reducción en el grado de deficiencias y limitaciones.

Palabras clave: Miastenia Gravis; Fisioterapia; Pediatría; Informe de un caso

Trabalho realizado no Centro Universitário Jorge Amado (UNIJORGE), Salvador-BA, Brasil.

Conflito de interesse: não

Recebido em: 09/03/2021

Aceito em: $26 / 10 / 2021$

Endereço para correspondência: Michelli Christina Magalhães Novais. Centro Universitário Jorge Amado. Av. Luís Viana Filho 6775. São Marcos. CEP 41680-400. Salvador-BA, Brasil. E-mail: novaismichelli@outlook.com

\section{INTRODUÇÃO}

\section{A miastenia gravis é uma doença neuromuscular} autoimune, de origem genética, que causa a inibição da atividade sináptica na junção neuromuscular através da ação de anticorpos nos receptores nicotínicos de acetilcolina, resultando em disfunção motora ${ }^{1,2}$. Quando a apresentação dos sintomas ocorre em indivíduos menores de 19 anos, é denominada miastenia gravis juvenil (MGJ)2 . 
A MGJ é considerada rara, com estimativa mundial de aproximadamente 1 a 5 casos por ano, a cada 1 milhão de pessoas $^{2}$, com frequência correspondente a $10 \%$ do total dos casos de miastenia gravis ${ }^{3}$.

Muitas crianças e adolescentes com MGJ podem tornar-se dependentes de tecnologias médicas, caracterizando uma Condição Crônica Complexa (CCC) ${ }^{4}$, que pode necessitar de suporte específico para a transição do ambiente hospitalar ao domiciliar. Essa transição pode ser realizada por setores especializados, como a Unidade de Treinamento para Desospitalização (UTD), onde ocorre a preparação da criança e seus cuidadores para o Programa de Assistência Domiciliar (PAVD) ${ }^{5}$.

Na UTD crianças e adolescentes recebem assistência integral, em vista de suas necessidades clínicas, funcionais e psicossociais. Além disso, seus cuidadores/familiares são submetidos a treinamento multidisciplinar, visando a desospitalização segura e com manutenção dos cuidados ${ }^{5}$. A equipe desta unidade é composta por profissionais da enfermagem, fonoaudiologia, psicologia, terapia ocupacional, assistência social, nutrição, medicina e fisioterapia. Além das peculiaridades do amparo da UTD, quanto às atribuições fisioterapêuticas na MGJ, destacamse, a prevenção de incapacidades secundárias à doença, bem como a promoção da restauração dos desvios de função ${ }^{6,7}$.

As deficiências consequentes da MGJ ocorrem devido aos comprometimentos da força dos músculos periféricos, 
respiratórios e da deglutição, que implicam em limitações na realização de atividades de vida diária ${ }^{8}$. Entretanto, há uma carência de estudos que descrevem as repercussões da MGJ não somente na atividade, mas também na participação, o que ressalta a relevância desta investigação. Além disso, é importante compreender a atuação fisioterapêutica no preparo à desospitalização de crianças com MGJ, pois o melhor entendimento acerca de tais aspectos pode auxiliar na proposição de intervenções fisioterapêuticas diferenciadas, com foco na capacidade e desempenho. Deste modo, o objetivo deste estudo é descrever a atuação fisioterapêutica e a evolução funcional de uma criança com diagnóstico de MGJ.

\section{MÉTODO}

Trata-se de um estudo observacional, descritivo e retrospectivo: relato de caso. Este estudo foi aprovado pelo Comitê de Ética e Pesquisa da Maternidade Climério de Oliveira, sob o parecer número 3.852.803. A responsável pela criança assinou o Termo de Consentimento Livre e Esclarecido e o Termo de Autorização Para Uso e Veiculação de Imagem.

Foi descrito o caso clínico, com enfoque na atuação fisioterapêutica e desfecho funcional, de uma criança com diagnóstico de MGJ, assistida em um hospital pediátrico de referência. As informações utilizadas foram oriundas de registros em prontuários realizados pela equipe multiprofissional de saúde. Foram colhidos dados como a 
data, período e setor de internação, desfechos clínicos, procedimentos fisioterapêuticos e desfechos funcionais.

Os dados sobre a funcionalidade foram obtidos através de registros da Functional Status Scale (FSS) pediátrica. Esta escala é utilizada para verificar o estado funcional de crianças que foram submetidas à hospitalização. A FSS é composta pelos seguintes domínios: estado mental, funcionamento sensorial, comunicação, funcionamento motor, estado respiratório e alimentação. A partir da aplicação deste instrumento, a criança pode ser classificada com função adequada, disfunção leve, disfunção moderada, disfunção grave ou disfunção muito grave ${ }^{9}$.

\section{RELATO DE CASO}

Criança MRS, sexo feminino, 7 anos, admitida em uma Unidade de Pronto Atendimento de Salvador, Bahia, no dia sete de maio de 2018, com relato de perda ponderal. Durante a avaliação apresentou sinais de hipoatividade, diminuição na frequência respiratória, frequência cardíaca e saturação periférica de oxigênio, evoluindo para um quadro de choque séptico e insuficiência respiratória aguda, sendo necessária a intubação orotraqueal. Foi transferida para a Unidade de Terapia Intensiva (UTI) de um hospital pediátrico filantrópico da cidade, tendo diagnóstico de pneumonia comunitária. $\mathrm{Na}$ anamnese familiar foi obtido o relato de perda ponderal de $7 \mathrm{~kg}$ no mês que antecedeu a internação, além de sinais de fraqueza muscular e tosse recorrente. 
Na UTI, permaneceu em ventilação mecânica, apresentando quadro de Síndrome do Desconforto Respiratório Agudo de classificação moderada (índice de oxigenação de 14 pontos), cursando com duas falhas na extubação devido à falência ventilatória, entre os dias 12 a 21 de maio de 2018.

Devido à suspeita de MGJ, foi iniciado teste terapêutico com piridostigmina. Durante este período, fez uso de imunoglobulina por orientação da equipe de neurologia, sendo traqueostomizada no dia 4 de junho, após tentativas de extubação e desmame dos parâmetros ventilatórios.

Durante a internação na UTI, recebeu intervenções fisioterapêuticas com enfoque respiratório e motor. Enquanto sedada, foi submetida a procedimentos como cinesioterapia passiva, alongamentos, mobilização articular e posicionamento no leito, e cuidados respiratórios (terapia de higiene brônquica, ventilação não-invasiva após as extubações e terapia de expansão pulmonar). Quando consciente, realizou cinesioterapia ativa-livre, transferências no leito, ortostase e treino de marcha. Além disso, mesmo em ventilação mecânica, deambulou com apoio por curta distância na UTI.

Em 19 de junho de 2018 foi transferida para o setor UTD para início da desospitalização, dependente de ventilação mecânica invasiva, via traqueostomia, deambulando curtas distâncias com apoio unilateral. Apresentava redução de força dos músculos dos membros 
inferiores, mímica facial, musculatura respiratória (pressões inspiratória e expiratória máximas não avaliadas devido a não colaboração da paciente) e ptose palpebral. Além disso, apresentava fraqueza dos músculos da deglutição, sendo necessária sonda nasogástrica para alimentação.

Apesar da suspeita diagnóstica de MGJ, acreditava-se que os sinais de fraqueza muscular foram consequências da redução de mobilidade durante o internamento. Todavia, a persistência dessas desordens corroborou a suspeita de doença neuromuscular. A confirmação diagnóstica de MGJ ocorreu no início de setembro de 2018, resultado do exame Anti Musk positivo, valor encontrado $0,92 \mathrm{nmol} / \mathrm{L}$ (valor de normalidade inferior a $0,05 \mathrm{nmol} / \mathrm{L})^{10}$. A partir do diagnóstico, houve ajuste do tratamento farmacológico, passando a utilizar, além da piridostigmina, a azatioprina e prednisolona.

$\mathrm{Na}$ UTD, as atividades de intervenções fisioterapêuticas eram realizadas de forma lúdica envolvendo os profissionais da equipe multiprofissional, como da terapia ocupacional e fonoaudiologia (Figura 1 $A / B$ ). Esta abordagem foi necessária, visto que a criança apresentava humor oscilante, com momentos de rebaixamento, possivelmente pelo longo período de internação, uso de dispositivos como traqueostomia, sondas e ventilação mecânica invasiva. Para estimular a participação social, foram realizadas saídas do espaço físico da UTD (Figura 2 A/B), como idas à brinquedoteca e deambulação por outros ambientes do hospital. Além de 
atividades de musicalização e estímulo às atividades de vida diária.

Figura 1. A: Treino para controle de tronco. B: Intervenção com ludicidade.

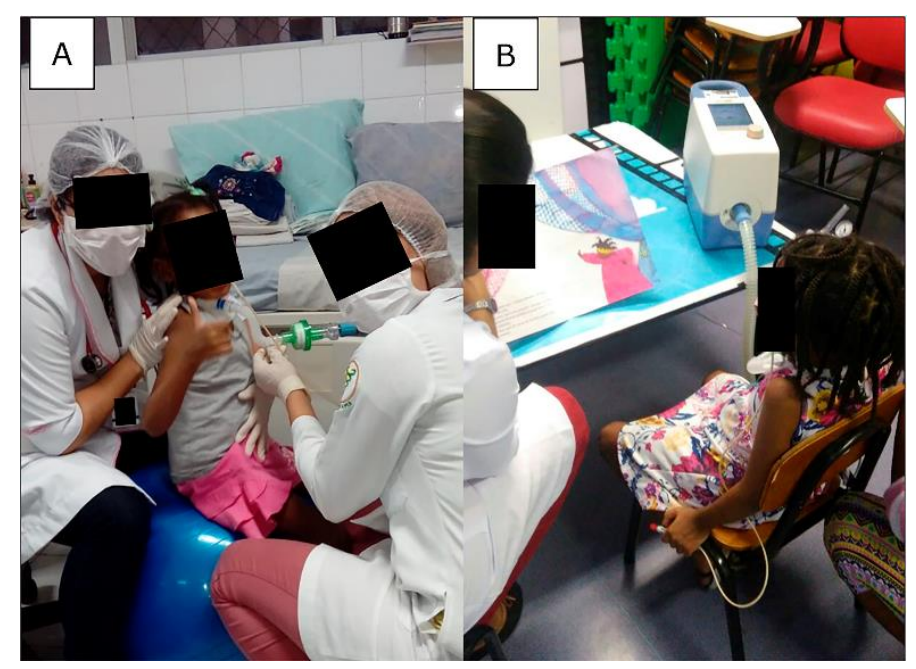

Figura 2. A: Passeio externo (ao shopping). B: Treino de marcha em ambiente externo.

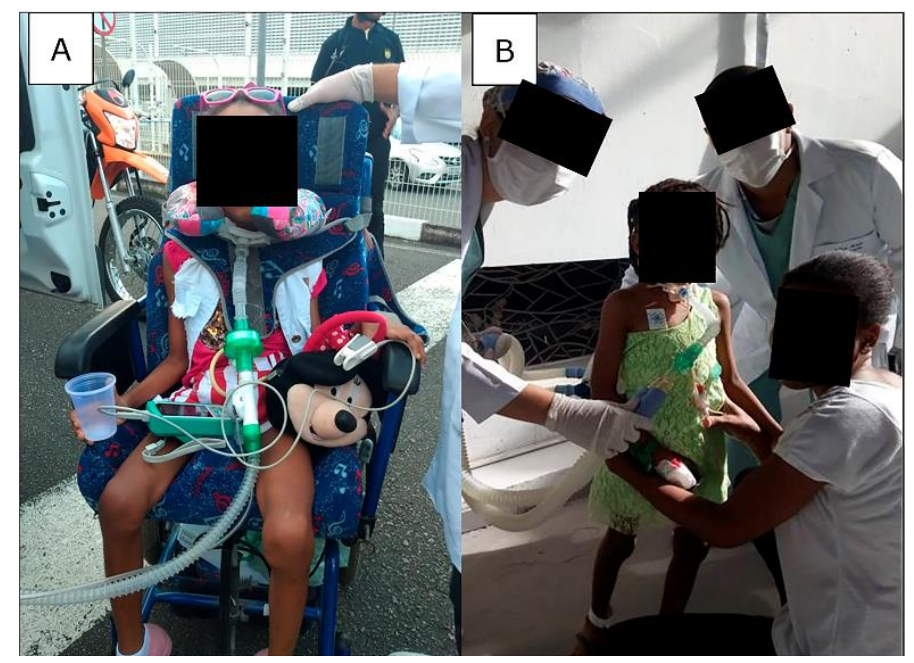


Durante a estadia na UTD, em um período de 3 semanas, a paciente apresentou progressos no quadro motor: realizou ortostatismo independente e deambulou com suporte unilateral, subiu e desceu escadas com apoio. Quanto ao quadro respiratório, não houve evolução no desmame do suporte ventilatório mecânico invasivo. A criança permaneceu em modo Ventilação Mandatória Intermitente Sincronizada, não tolerando a mudança para modo Ventilação por Pressão de Suporte. As tentativas de desmame falharam devido à persistência de quadros de desconforto respiratório, hipercapnia e atelectasia, além da incapacidade dos músculos respiratórios em manter ventilação espontânea.

Ao longo do tratamento a criança não obteve melhora no quadro de fraqueza nos músculos da deglutição, resultando na realização de gastrostomia, em seis de janeiro de 2019. Após 30 dias teve alta da UTD, passando a receber, em domicílio, o suporte do PAVD (Figura 3 A/B). A criança seguiu acompanhada por equipe multiprofissional, realizando reabilitação em casa.

A paciente deste estudo, no momento da admissão na UTD, apresentou 18 pontos na FSS caracterizando uma disfunção grave. Enquanto na alta, apresentou 15 pontos na escala (disfunção moderada), demonstrando evolução no seu estado funcional (Tabela 1 ). 
Figura 3. Paciente em ambiente domiciliar.

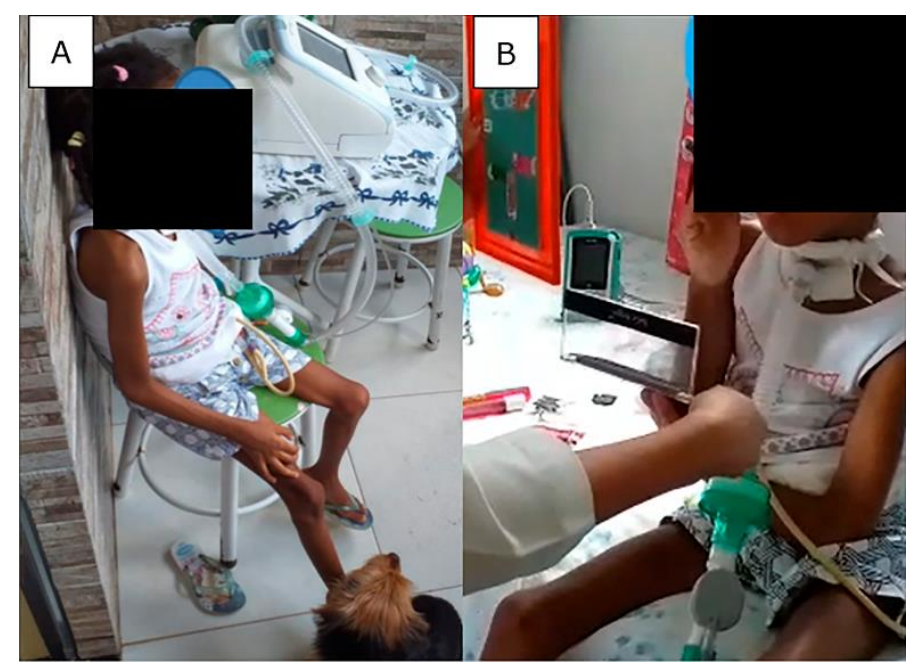

Tabela 1. Aplicação da escala Functional Status Scale (FSS).

\begin{tabular}{lcc}
\hline Momento/função & ADM na UTD & Alta da UTD \\
\hline Estado mental & 2 & 1 \\
\hline Funcionalidade sensorial & 2 & 1 \\
\hline Comunicação & 2 & 2 \\
\hline Funcionamento motor & 4 & 3 \\
\hline Alimentação & 3 & 3 \\
\hline Estado Respiratório & 5 & 5 \\
\hline Total & $\mathbf{1 8}$ & $\mathbf{1 5}$ \\
\hline ADM = admissão; UTD = Unidade de Treinamento para Desospitalização.
\end{tabular}

\section{DISCUSSÃO}

Este estudo descreve a atuação fisioterapêutica em uma doença rara, promovida em um dos poucos setores do Brasil especializados em treinamento para desospitalização de crianças e adolescentes com condição crônica complexa. 
Os distúrbios decorrentes da MGJ podem evoluir de forma crítica, necessitando de cuidados intensivos ${ }^{11}$, principalmente quando há necessidade de suporte ventilatório mecânico ${ }^{12,13}$. Neste contexto, falhas sucessivas de extubação podem indicar a realização da traqueostomia, uma vez que esse dispositivo pode facilitar a transição para a ventilação espontânea, como se deu com a criança do presente relato de caso. O processo de desmame da ventilação mecânica de pacientes com MGJ é complexo, devido a comum progressão rápida da fatigabilidade dos músculos ventilatórios ${ }^{11}$. A fisioterapia atua frente a essas complicações, no manejo e monitorização do quadro respiratório ${ }^{14}$.

Quanto ao enfoque nas disfunções motoras, devido à paciente apresentar o cognitivo preservado, a intervenção fisioterapêutica foi facilitada pela musicalização e ludicidade utilizadas nas intervenções, como estratégia para lidar com episódios de recusa ao atendimento, decorrente de algumas oscilações de humor. A utilização de música em intervenções terapêuticas pode auxiliar na regulação de tensão psicológica, humor e motivação ${ }^{15}$. Além disso, a ludicidade torna os atendimentos mais prazerosos, consistindo em artifícios para potencializar o efeito terapêutico ${ }^{16}$.

Em pacientes adultos com miastenia gravis é sabido que a fisioterapia pode promover aumento da capacidade física, funcionalidade e equilíbrio ${ }^{17}$. 0 tratamento fisioterapêutico realizado na UTD proporcionou melhorias 
funcionais para a criança deste relato, que evoluiu de uma disfunção grave para moderada, segundo a FSS.

No que se refere ao suporte do quadro respiratório, a mudança do ventilador mecânico multiprocessado para o portátil promoveu maior possibilidade de mobilidade. Entretanto, devido ao fato das complicações motoras da MGJ serem oscilantes, houve episódios de declínio da força muscular ${ }^{3}$, representando um desafio na adequação da intervenção fisioterapêutica. Ressalta-se que, o tratamento da MGJ envolve também o uso de fármacos que podem causar importantes efeitos colaterais ${ }^{18}$. Deste modo, além das particularidades da MGJ, o uso de medicamentos direcionados ao controle da progressão desta doença também pode proporcionar oscilação do desempenho físico funcional ${ }^{19}$.

De maneira geral, pode-se perceber que as particularidades da UTD proporcionaram um novo contexto para a participação social, por ser um ambiente mais amplo e que favorece maior convivência com outras crianças, bem como mais interação com os profissionais da equipe multiprofissional, pois estes também promovem 0 treinamento de seus cuidadores.

No que se refere ao contexto domiciliar, recebendo o suporte do PAVD, é razoável supor que a criança foi proporcionada a um ambiente que pode promover maior sensação de estabilidade, pois há maior interação e estímulo à participação social, devido à liberdade de convívio com a família no ambiente domiciliar ${ }^{20}$. 
Quanto ao diagnóstico da MGJ, uma condição que gera deficiência de força muscular e consequente limitação para a capacidade e restrições do desempenho ${ }^{8}$, conhecer suas repercussões clínicas e cinético funcionais pode permitir um melhor direcionamento para a criação de protocolos fisioterapêuticos que se adaptem às características da doença.

Este estudo apresenta limitações como a impossibilidade da avaliação de outros desfechos de forma quantitativa, como a mensuração da força muscular respiratória, através da obtenção dos valores de pressões inspiratória e expiratória máximas. Apesar disso, aspectos relevantes foram descritos, como o estado funcional e a força muscular periférica da criança.

\section{CONCLUSÃO}

A assistência fisioterapêutica recebida pela criança neste relato de caso, foi pautada na promoção de atividade e participação. Além disso, a ludicidade foi uma característica fundamental do atendimento fisioterapêutico, permeando a melhor adesão às intervenções propostas, alcançando-se, deste modo, resultados como a minimização do grau de deficiências de função e limitações de capacidades. Apesar da evolução no seu estado funcional, algumas disfunções motoras e respiratórias persistiram. Todavia, estas não impossibilitaram a desospitalização. 


\section{AGRADECIMENTOS}

Agradecemos ao Centro Universitário Jorge Amado (UNIJORGE) o incentivo à pesquisa, através da Iniciação Científica, bem como à Maiara Lanna Souza Bacelar Bouzas pelo estímulo à realização deste estudo.

\section{REFERÊNCIAS}

1.Carr AS, Cardwell CR, McCarron PO, McConville J. A systematic review of population based epidemiological studies in Myasthenia Gravis. BMC Neurol 2010;10:46. https://doi.org/10.1186/1471-2377$10-46$

2.Peragallo JH. Pediatric Myasthenia Gravis. Semin Pediatr Neurol 2017;24:116-21. https://doi.org/10.1016/j.spen.2017.04.003

3. Hehir MK, Silvestri NJ. Generalized Myasthenia Gravis: Classification, Clinical Presentation, Natural History, and Epidemiology. Neurol Clin 2018;36:253-60. https://doi.org/10.1016/j.ncl.2018.01.002

4.Cohen E, Kuo DZ, Agrawal R, Berry JG, Bhagat SKM, Simon TD, et al. Children with medical complexity: An emerging population for clinical and research initiatives. Pediatrics 2011;127:529-38. https://doi.org/10.1542/peds.2010-0910

5. Novais MCM, Victor DS, Rodrigues DS, Freitas BO, Barreto NMPV, Mendes DJS, et al. Factors associated with de-hospitalization of children and adolescents with complex chronic condition. Rev Paul Ped 2021;39:1-7. $\quad$ https://doi.org/10.1590/19840462/2021/39/2020118

6. Eagle M. Report on the Muscular Dystrophy Campaign workshop: Exercise in neuromuscular diseases Newcastle, January 2002. https://doi.org/10.1016/s0960-8966(02)00136-0

7.Farrugia ME, Goodfellow JA. A Practical Approach to Managing Patients With Myasthenia Gravis - Opinions and a Review of the Literature. Front Neurol 2020;11:1-16. https://doi.org/10.3389/fneur.2020.00604

8. Marina AD, Trippe H, Lutz S, Schara U. Juvenile Myasthenia Gravis : Recommendations for Diagnostic Approaches and Treatment. Neuropediatrics 2014;45:75-83. https://doi.org/10.1055/s-00331364181

9.Bastos VCS, Carneiro AAL, Barbosa MSR, Andrade LB. Brazilian version of the Pediatric Functional Status Scale: Translation and cross-cultural adaptation. Rev Bras Ter Intensiva 2018;30(3):301-7. https://doi.org/10.5935/0103-507X.20180043

10.Kostera-Pruszczyk A, Kwiecinski H. Juvenile seropositive myasthenia gravis with anti-MuSK antibody after thymectomy. J 
https://doi.org/10.1007/s00415-009-5215-2

11.Damian MS, Srinivasan R. Neuromuscular problems in the ICU. Curr Opin Neurol 2017;30:538-44. https://doi.org/10.1097/wco.0000000000000480

12. Hogan CD, Lee J, Sleigh BC, Banerjee PR, Ganti L. Acute Myasthenia Crisis: A Critical Emergency Department Differential. Cureus 2020;12:8-13. https://dx.doi.org/10.7759\%2Fcureus.9760

13.Piastra M, Conti G, Caresta E, Tempera A, Chiaretti A, Polidori G, et al. Noninvasive ventilation options in pediatric myasthenia gravis. Paediatr Anaesth 2005;15:699-702. https://doi.org/10.1111/j.14609592.2005.01617.x

14.Lizarraga AA, Lizarraga KJ, Benatar M. Getting Rid of Weakness in the ICU: An Updated Approach to the Acute Management of Myasthenia Gravis and Guillain-Barré Syndrome. Semin Neurol 2016;36:615-24. http://dx.doi.org/10.1055/s-0036-1592106

15.Stegemann T, Geretsegger M, Phan Quoc E, Riedl H, Smetana M. Music Therapy and Other Music-Based Interventions in Pediatric Health Care: An Overview. Medicines 2019;6:25. https://doi.org/10.3390/medicines6010025

16.Silva AS, Valenciano PJ, Fujisawa DS. Atividade Lúdica na Fisioterapia em Pediatria: Revisão de Literatura TT - Playing in Pediatric Physical Therapy: Literature Review. Rev Bras Educ Espec 2017;23:623-36. http://dx.doi.org/10.1590/s141365382317000400011

17.Corrado B, Giardulli B, Costa M. Evidence-based practice in rehabilitation of myasthenia gravis. A systematic review of the literature. J Funct Morphol Kinesiol 2020;5:71. https://doi.org/10.3390/jfmk5040071

18.Finnis MF, Jayawant S. Juvenile Myasthenia Gravis: A Paediatric Perspective. Autoimmune Dis 2011;404101. https://doi.org/10.4061/2011/404101

19.Sanders DB, Massey JM. Clinical features of myasthenia gravis. Handb Clin Neurol 2008;91:243-61. https://doi.org/10.1016/S00729752(07)01507-2

20.Carnevale FA, Alexander E, Davis M, Rennick J, Troini R. Daily living with distress and enrichment: The moral experience of families with ventilator-assisted children at home. Pediatrics 2006;117:e4860. https://doi.org/10.1542/peds.2005-0789 